\title{
Reproducción de los róbalos Centropomus undecimalis y C. ensiferus (Perciformes: Centropomidae) en una laguna costera tropical
}

\author{
Juan Gassman ${ }^{1}$, Héctor López Rojas ${ }^{2} \&$ Dana Padrón ${ }^{3}$ \\ 1. Postgrado en Zoología, Instituto de Zoología y Ecología Tropical, Universidad Central de Venezuela, Caracas 1040, \\ Venezuela; gassman.j@gmail.com \\ 2. Laboratorio de ictiología, Instituto de Zoología y Ecología Tropical, Universidad Central de Venezuela, Caracas 1040, \\ Venezuela; hector.lopez@ciens.ucv.ve \\ 3. Facultad Experimental de Ciencias, Universidad del Zulia, Maracaibo 526, Venezuela; danapadron@gmail.com
}

Recibido 28-III-2016. Corregido 09-IX-2016. Aceptado 11-X-2016.

\begin{abstract}
Reproduction of Centropomus undecimalis and C. ensiferus (Perciformes: Centropomidae) in a tropical coastal lagoon. Snooks $C$. undecimalis and $C$. ensiferus are important fishery resources in their distribution area. In Venezuela they are caught by artisanal fisheries along the entire coast. However, studies on their biology that would help establish fishing regulations are scarce. To study reproduction aspects of these species, we analyzed 1325 individuals of $C$. undecimalis and 2025 of $C$. ensiferus caught by artisanal fishing in the Laguna de Tacarigua in Northern Venezuela, from July 2009 to September 2010. Total length, total weight, sex and stage of gonadal development were recorded. The sex ratio varied throughout the year: there was a significant difference in favor of $C$. undecimalis males (1.5:1), and C. ensiferus females (1:3.3). Differences were also evident in the sexual proportion at each size group. For both species, males dominated in the lower size ranges while females dominated in the larger size ranges. These results suggested sequential hermaphroditism (protrandry) in both species. Size at sexual maturity $\left(\mathrm{Lm}_{50}\right)$ for $C$. undecimalis was $\phi=73.6$ and $\hat{\gamma}=59.3 \mathrm{~cm}$, and $\phi=26$ and $\delta=21.5 \mathrm{~cm}$ for $C$. ensiferus. $C$. ensiferus reproduced throughout the year with two periods of higher intensity (August to September and February to April). Although we found very few mature individuals for $C$. undecimalis, February showed an important reproduction peak. Growth differences were found between males and females of $C$. undecimalis: positive allometry for females $\left(\mathrm{P}=0.0022 * \mathrm{~L}^{3.353}\right)$ and negative allometry for males $\left(\mathrm{P}=0.0108^{*} \mathrm{~L}^{2.945}\right)$. Both males and females of $C$. ensiferus showed a positive allometric relationship $\left(\mathrm{P}=0.0049 * \mathrm{~L}^{3.235}\right)$. Lack of fishing regulations and controls, evidenced in this study by significant volumes of immature individuals in the catches, and the particular reproductive biology of these species, strongly indicates the need to establish regulations to ensure their responsible use. We recommend establishing a closed season during the first quarter of the year and minimum catch sizes for both species. Rev. Biol. Trop. 65 (1): 181-194. Epub 2017 March 01.
\end{abstract}

Key words: protandric hermaphroditism, Centropomus, snook, Laguna de Tacarigua.

Centropomus undecimalis (Bloch, 1792) y C. ensiferus Poey, 1860, conocidos comúnmente como róbalos, son peces demersales, frecuentes en zonas estuarinas y lagunas costeras. Poseen una distribución tropical y subtropical en la costa occidental del Océano Atlántico desde el sureste de Florida, en los Estados Unidos hasta el sur de Brasil (Rivas, 1986; Cervigón, 1991). Se caracterizan por un ciclo de vida diádromo, la reproducción se lleva a cabo en ambientes marinos, las larvas y juveniles migran hacia estuarios y ríos, que usan como áreas de criadero en donde permanecen generalmente hasta ser subadultos o alcanzar la madurez sexual (Peters, Matheson, \& Taylor, 1998; Taylor, Whittington, Grier, \& Crabtree, 2000).

Todas las especies del género son aprovechadas por la pesca, aunque $C$. undecimalis es una de las que soporta las mayores pesquerías 
comerciales en su área de distribución, por el tamaño que alcanza y la calidad de su carne, así mismo, es de gran interés para la pesca deportiva y recreativa (Taylor et al., 2000; Perera-García et al., 2013). C ensiferus por su parte, conocido también como tripón, es la especie más pequeña del género, considerada como descarte en algunas pesquerías de arrastre de camarón (Chao et al., 2015). Sin embargo, en Venezuela ambas son aprovechadas a lo largo de la costa, particularmente en estuarios como el Lago de Maracaibo o el delta del Río Orinoco y en lagunas costeras, donde son parte de una importante extracción comercial por los pescadores locales durante todo el año (Cervigón et al., 1992; Gassman \& López, 2015a). A pesar de esto, no existe en el país ninguna regulación para su captura y son escasos los estudios que se han llevado a cabo sobre estas especies.

La determinación de parámetros reproductivos son considerablemente importantes, como insumos en el manejo y gestión de pesquerías de poblaciones explotadas de peces (Jennings, Kaiser, \& Reynolds, 2001; Froese, 2004). Numerosos estudios previos sobre la reproducción de $C$. undecimalis han sido realizados en el área del Caribe, señalando que la especie posee reproducción múltiple o por tandas, y exhibe un hermafroditismo protándrico, en el cual la mayoría de los individuos jóvenes son machos y a partir de un determinado tamaño inician una reversión sexual (Peters et al., 1998; Grier \& Taylor, 1998; Taylor, Grier, \& Whittington, 1998; Taylor et al., 2000; Adams, Wolfe, Barkowski, \& Overcash, 2009; Andrade, Santos, \& Taylor, 2013; Lowerre-Barbieri et al., 2014). En contraste, sobre C. ensiferus son escasos los antecedentes, más allá de algunos aspectos biológicos presentes en los trabajos realizados por Márquez (1985), Keith, Le Bail \& Planquette (2000) y Sánchez (2001).

Ambas especies están consideradas por la Unión Internacional para la Conservación de la Naturaleza (UICN) en estado de preocupación menor, debido a que no hay evidencia de un declive significativo de sus poblaciones, aunque hay indicios de sobrepesca de
C. undecimalis en regiones de Florida (Estados Unidos) y el Golfo de México (Perera-García et al., 2013; Mendonça et al., 2015, mientras el estatus poblacional de $C$. ensiferus se considera desconocido (Chao et al., 2015). En este sentido, el presente trabajo tuvo como objetivo generar información sobre aspectos reproductivos de C. undecimalis y C. ensiferus que contribuyan al conocimiento de su biología reproductiva y al establecimiento de medidas para su aprovechamiento responsable.

\section{MATERIALES Y MÉTODOS}

Área de estudio: El presente trabajo se llevó a cabo en la laguna costera de Tacarigua, Parque Nacional (1974) y sitio Ramsar (1996), ubicada al sur del mar Caribe en la costa central de Venezuela $\left(10^{\circ} 11^{\prime} 30^{\prime \prime}-10^{\circ} 20^{\prime} 20^{\prime \prime} \mathrm{N} \&\right.$ $\left.65^{\circ} 41^{\prime} 10^{\prime \prime}-65^{\circ} 57^{\prime} 20^{\prime \prime} \mathrm{W}\right)$. Posee alrededor de 7800 ha, está separada del mar por una barra litoral de unos $28.8 \mathrm{~km}$ y una sola boca de comunicación con el mar de variable amplitud y en algunas épocas completamente cerrada. La profundidad es en promedio de $1.2 \mathrm{~m}$ con una amplitud máxima de marea diurna de $0.30 \mathrm{~m}$. El clima de la zona es semiárido, con una temperatura media de $26.5^{\circ} \mathrm{C}$ y con una precipitación anual menor de 1000 mm (Ramírez, 1996; Novo, Morales, Rodríguez, Martínez, \& De Hertelendy, 1997). Durante el presente estudio, la laguna se mantuvo sin comunicación con el mar por nueve meses (septiembre 2009 a mayo 2010), producto de un intenso periodo de sequía hasta abril 2010, cuando inició un fuerte periodo de lluvias, que se prolongó hasta el mes de julio del mismo año. Las Temperaturas variaron ampliamente entre las diferentes zonas de la laguna $\left(27.4\right.$ a $\left.33.4{ }^{\circ} \mathrm{C}\right)$ al igual que la salinidad (7.2 a $24.3 \mathrm{ppm}$ ).

Se realizaron muestreos mensuales de individuos provenientes de la pesca artesanal de los puertos de desembarque adyacentes a la laguna (Tacarigua, Paparo, San Ignacio, Machurucuto y Las Lapas) desde julio 2009 a septiembre 2010, capturados con atarrayas y redes de ahorque, de abertura de malla de entre 1.5 a $9 \mathrm{~cm}$ y 7.5 a $12 \mathrm{~cm}$, respectivamente. 
Los ejemplares se examinaron in situ midiendo la longitud total $(\mathrm{cm})$ (ictiómetro Wildco), el peso total (g) (balanza Ohaus), se identificó el sexo y el grado de madurez sexual según el criterio de Nikolski (1963) simplificado por Andrade et al. (2013) de cuatro clases: inmaduro, en desarrollo, maduro y desovado.

Para evaluar diferencias significativas en la proporción sexual mensual y por intervalos de tallas, se aplicó la prueba Chi-cuadrada $\left(\chi^{2}\right.$, $\mathrm{p}<0.05)$ (Zar, 1996). Se estimó la talla media de madurez sexual del $50 \%$ de la población $\left(\mathrm{Lm}_{50}\right)$ de la fracción de ejemplares maduros (clases maduros y desovados) en cada intervalo de talla $\left(\mathrm{FM}_{(\mathrm{L})}\right)$ mediante la ecuación de la curva logística $\mathrm{FM}_{(\mathrm{L})}=1 / 1+\mathrm{e}^{(\mathrm{S} 1-\mathrm{S} 2 * \mathrm{~L})}$ (Hoydal, Rorvik, \& Sparre,1982), donde, L es la marca de clase; $\mathrm{S}_{1}$ y $\mathrm{S}_{2}$ se calcularon con la ecuación de la regresión lineal: $\operatorname{Ln}\left(1 / \mathrm{FM}_{(\mathrm{L})}-1\right)=$ $\mathrm{S}_{1}-\mathrm{S}_{2} * \mathrm{~L}$, donde, $\mathrm{F}_{(\mathrm{L})}$ es la fracción a cada talla; L es la marca de Clase; $\mathrm{S}_{1}=$ a y $\mathrm{S}_{2}=-\mathrm{b}$ (Gaertner \& Laloé, 1986).

La relación longitud-peso se obtuvo mediante la ecuación de Ricker (1975): $\mathrm{P}=$ $\mathrm{a}^{*} \mathrm{~L}^{\mathrm{b}}$, donde, $\mathrm{P}$ es el peso total (g); L es la longitud total $(\mathrm{cm})$; a es el intercepto del eje de las ordenadas y b es la pendiente de la curva. Se realizó una prueba t-student para determinar la existencia de diferencia significativa entre sexos (Zar, 1996). El factor o estado de condición se estimó por el índice de Fulton: $\mathrm{K}=$ $100\left(\mathrm{P} / \mathrm{L}^{3}\right)$ (Froese, 2006), mediante el ANDEVA de una vía, se analizó la variación mensual y por sexos de $\mathrm{K}$.

\section{RESULTADOS}

En las capturas totales de la Laguna de Tacarigua durante el periodo de estudio, los róbalos representaron el $12.5 \%$ de ellas. Fueron analizados un total de 1351 ejemplares de C. undecimalis con tallas comprendidas entre 17 y $108 \mathrm{~cm}$ de longitud total (Fig. 1A) y peso total entre 70 y $10800 \mathrm{~g}$. Del total, 457 fueron hembras (33.8\%), 706 machos (52.3\%) y 188 de sexo indeterminado (13.9\%). La longitud de las hembras varió entre 37 y $108 \mathrm{~cm}$, con un promedio de $54.9 \mathrm{~cm}$, mientras que los machos estuvieron entre 17 y $74 \mathrm{~cm}$, con un promedio de $41.6 \mathrm{~cm}$.

De C. ensiferus se examinaron 2025 ejemplares con tallas entre 16.5 y $42.5 \mathrm{~cm}$ de longitud total (Fig. 1B) y pesos que variaron de 25.1 a 820 g. De este total, 1555 fueron hembras $(76.8 \%)$ y 470 machos $(23.2 \%)$. La longitud total de las hembras osciló entre 18.6 y $42.5 \mathrm{~cm}$, con un promedio de $25.8 \mathrm{~cm}$, mientras que en los machos fluctuó entre 16.5 y $26.5 \mathrm{~cm}$, con un promedio de $21.3 \mathrm{~cm}$ (Fig. 1B).

Proporción sexual: El porcentaje de machos: hembras de $C$. undecimalis fue de 1.5:1, el cual varió a lo largo del periodo de muestreo (Fig. 2A). No hubo diferencias en la proporción para los meses de julio, agosto, septiembre, febrero, marzo, abril y mayo. En C. ensiferus el porcentaje de machos y hembras también varió a lo largo del año (Fig. 2B), quedando la proporción 1:3.3 machos: hembras (Cuadro 1).

Proporción sexual a la talla: La proporción sexual por rango de tallas en C. undecimalis (Fig. 3A) mostró diferencias significativas entre las tallas de 19 a $46 \mathrm{~cm}$ y de 67 a $85 \mathrm{~cm}$ $\left(\chi^{2}, \mathrm{p}<0.05\right)$, no así entre las tallas de 47 a 68 $\mathrm{cm}$ y mayores de $88 \mathrm{~cm}$ de longitud total $\left(\chi^{2}\right.$, $\mathrm{p}>0.05)$, destacándose que todos los individuos mayores de $74.5 \mathrm{~cm}$ fueron hembras. En el caso de $C$. ensiferus, se encontró diferencia significativa entre las tallas de 18 a $21 \mathrm{~cm}$ y de 23 a 33 y $35 \mathrm{~cm}\left(\chi^{2}, \mathrm{p}<0.05\right)$, y no entre las tallas de 16 a 17, 22 y las mayores de $36 \mathrm{~cm}$ de longitud total $\left(\chi^{2}, \mathrm{p}>0.05\right)$, siendo todos los ejemplares hembras para tallas mayores de $26.5 \mathrm{~cm}$ (Fig. 3B).

Frecuencia mensual de las fases de madurez sexual: En $C$. undecimalis la frecuencia de individuos inmaduros estuvo representada a lo largo de todo el periodo de muestreo y fue la de mayor proporción (61.4 $\%$ ), siendo superior a las restantes en los meses de noviembre a enero y de marzo a septiembre. La fase de individuos en desarrollo fue particularmente representativa entre los meses 


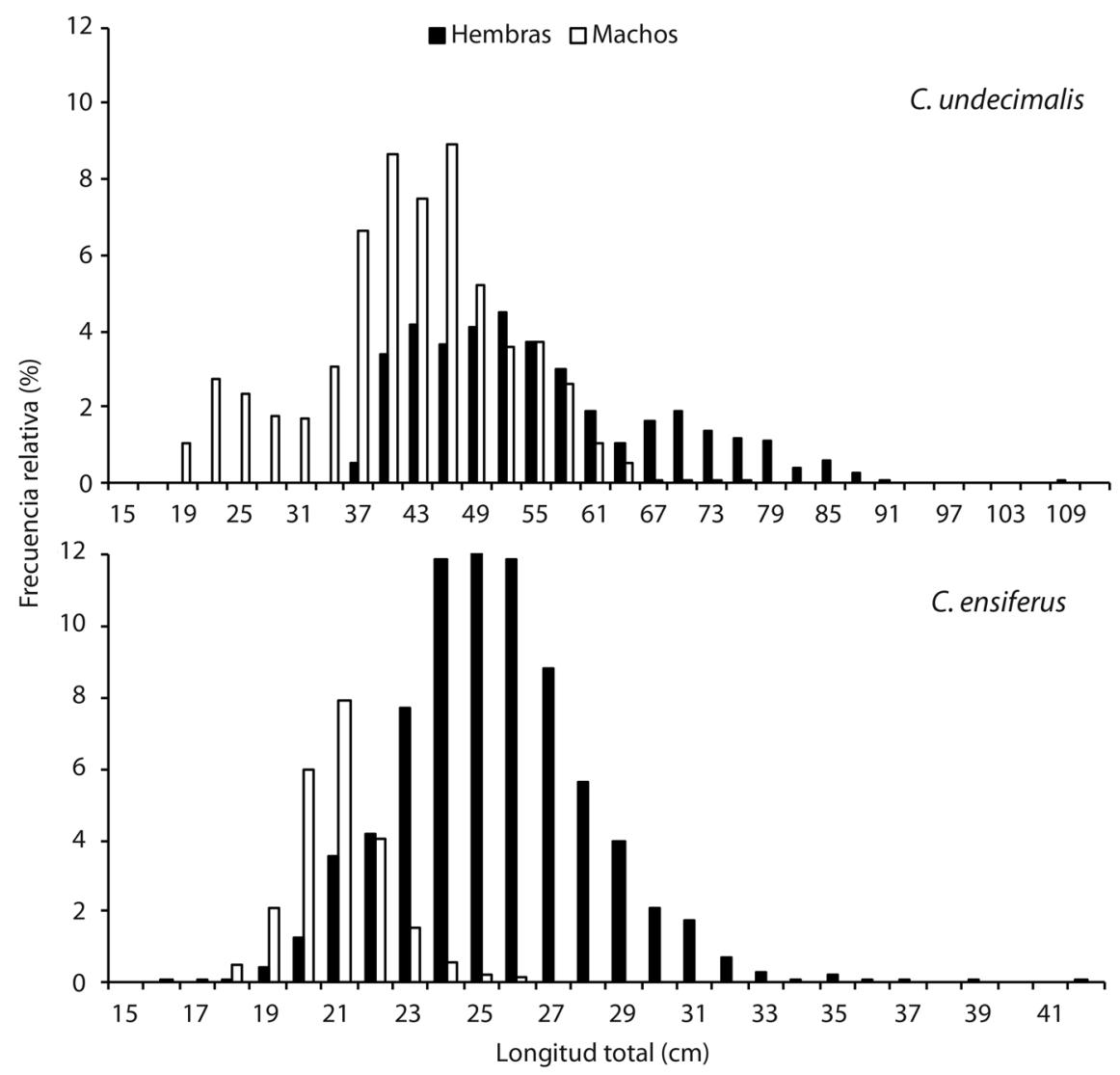

Fig. 1. Distribución de frecuencia de tallas de ejemplares de hembras y machos de C. undecimalis y C. ensiferus de julio 2009 a septiembre 2010 en la Laguna de Tacarigua, Venezuela.

Fig. 1. Distribution of lengths for male and females of C. undecimalis y C. ensiferus of July 2009 to September 2010 in the Laguna de Tacarigua, Venezuela.

de julio a diciembre, disminuyendo progresivamente el resto del periodo. Los ejemplares maduros (4.9\%), tanto hembras y machos, se presentaron principalmente durante el mes de febrero, la fase de desove estuvo muy poco representada (Fig. 4A).

Por el contrario, en C. ensiferus, aún cuando la frecuencia de individuos inmaduros estuvo presente a lo largo de todo el año, la misma estuvo poco representada $(7.3 \%)$, siendo su mayor frecuencia para los meses de junio a agosto de 2010. Los individuos en desarrollo fueron frecuentes durante todo el periodo de estudio (43.2\%), siendo mayoritarios de octubre a enero y de marzo a julio. Los individuos maduros $(46.7 \%)$ fueron abundantes en los meses agosto y septiembre de ambos años y febrero a abril, mostrando dos periodos de mayor intensidad reproductiva al año. Individuos desovados se encontraron en todos los meses con menor proporción (Fig. 4B).

Talla media de madurez sexual $\left(\mathrm{Lm}_{50}\right)$ : La talla media de madurez sexual de las hembras de $C$. undecimalis se determinó en $73.6 \mathrm{~cm}$ $\left(\mathrm{S}_{1}=11.57\right.$ y $\left.\mathrm{S}_{2}=0.16\right)$, mientras que para los machos fue de $59.3 \mathrm{~cm}\left(\mathrm{~S}_{1}=10.76\right.$ y $\left.\mathrm{S}_{2}=0.18\right)$ (Fig. 5A). En el caso de $C$. ensiferus, se encontró una talla de $26 \mathrm{~cm}$ para las hembras $\left(\mathrm{S}_{1}=\right.$ 20.56 y $\left._{2}=0.79\right)$ y $21.5 \mathrm{~cm}$ para los machos $\left(\mathrm{S}_{1}=34.64\right.$ y $\left.\mathrm{S}_{2}=1.61\right)$ (Fig. 5B).

Relación talla-peso y factor de condición: Para C. undecimalis se encontraron diferencias 
CUADRO 1

Proporción sexual mensual y tallas de captura de C. undecimalis y C. ensiferus de julio 2009 a septiembre 2010, en la Laguna de Tacarigua, Venezuela

TABLE 1

Monthly sex ratio and length catch of C. undecimalis and C. ensiferus during July 2009 to September 2010, in the Laguna de Tacarigua, Venezuela

\begin{tabular}{|c|c|c|c|c|c|c|c|c|c|c|}
\hline \multirow[b]{3}{*}{ Mes } & \multicolumn{5}{|c|}{ Centropomus undecimalis } & \multicolumn{5}{|c|}{ Centropomus ensiferus } \\
\hline & \multicolumn{2}{|c|}{$n$} & \multicolumn{3}{|c|}{ Talla (cm) } & \multicolumn{2}{|c|}{$n$} & \multicolumn{3}{|c|}{ Talla (cm) } \\
\hline & M & $\mathrm{H}$ & $\chi^{2}$ & Media & Min - Max & M & $\mathrm{H}$ & $\chi^{2}$ & Media & Min - Max \\
\hline Julio 2009 & 65 & 41 & $5.42 *$ & 35.3 & $17-80$ & 61 & 40 & $4.36^{*}$ & 24.3 & $17-37.5$ \\
\hline Agosto & 36 & 34 & 0.04 & 43.4 & $20.5-75$ & 21 & 183 & $128.64 *$ & 25.5 & $18.3-42.5$ \\
\hline Septiembre & 19 & 21 & 0.08 & 41 & $19.5-54.5$ & 13 & 271 & $234.38^{*}$ & 25.1 & $19.9-32.7$ \\
\hline Octubre & 95 & 69 & $4.11 *$ & 50.5 & $24.3-82.9$ & 9 & 86 & $62.4 *$ & 23.8 & $19.5-30$ \\
\hline Noviembre & 83 & 41 & $14.28 *$ & 45.6 & $23.4-76.2$ & 40 & 210 & $115.6^{*}$ & 24.7 & $19.4-31.7$ \\
\hline Diciembre & 89 & 44 & $15.22 *$ & 43.8 & $27.8-85.7$ & 12 & 93 & $62.48 *$ & 26.1 & $20.1-31.6$ \\
\hline Enero 2010 & 51 & 24 & $9.71 *$ & 50.7 & $34.3-83.6$ & 36 & 119 & $44.44 *$ & 25.5 & $18.7-33.1$ \\
\hline Febrero & 16 & 24 & 1.58 & 60.3 & $23.6-88.6$ & 25 & 161 & $99.44 *$ & 26.1 & $19.8-35.8$ \\
\hline Marzo & 62 & 46 & 2.36 & 43 & $23.1-77.6$ & 30 & 49 & $4.56^{*}$ & 24 & $16.5-33.2$ \\
\hline Abril & 27 & 29 & 0.05 & 53 & $30.5-87.5$ & 117 & 174 & $11.16^{*}$ & 24.1 & $18.5-31.7$ \\
\hline Mayo & 37 & 33 & 0.21 & 46.3 & $24.5-89.7$ & 32 & 65 & $11.22 *$ & 23.7 & $19.5-31$ \\
\hline Junio & 62 & 22 & $19.03 *$ & 44.2 & $35.1-108$ & 31 & 24 & 0.87 & 22.5 & $19.4-27.4$ \\
\hline Julio & 27 & 17 & 2.25 & 45.4 & $26.9-70.8$ & 36 & 16 & $7.67 *$ & 21.4 & $18.6-30$ \\
\hline Agosto & 18 & 11 & 1.66 & 48.3 & $37-60.1$ & 5 & 28 & $16^{*}$ & 27.6 & $20.7-39.5$ \\
\hline Septiembre & 19 & 1 & $16.15^{*}$ & 43.6 & $38.1-48.9$ & 2 & 36 & $30.39 *$ & 26 & $18.6-31.1$ \\
\hline Total & 706 & 457 & $53.31 *$ & 45.7 & $17-108$ & 470 & 1555 & $581.35^{*}$ & 24.8 & $16.5-42.5$ \\
\hline
\end{tabular}

* Proporción sexual significativamente diferente de $1: 1\left(\mathrm{p}<0.05, \chi^{2}=3.841\right) . n=$ número de ejemplares examinados; $\mathrm{M}=$ machos; $\mathrm{H}=$ hembras; Min= Valor mínimo; $\mathrm{Max}=$ valor máximo.

* Sex ratio significantly different $1: 1\left(\mathrm{p}<0.05, \chi^{2}=3.841\right) . n=$ number of specimens examined; $\mathrm{M}=$ males; $\mathrm{H}=$ females; Min= minimum value; $M a x=$ maximum value.

significativas en el crecimiento con respecto al peso entre machos $\mathrm{y}$ hembras $(\mathrm{t}=5.18$, g.l. $=$ $1169, \mathrm{p}<0.05$ ), quedando establecidas las siguientes relaciones, de tipo alométrico positivo para hembras $\left(\mathrm{P}=0.0022 * \mathrm{~L}^{3.353} ; \mathrm{R}^{2}=0.98\right)$ y negativo para machos $\left(\mathrm{P}=0.0108 * \mathrm{~L}^{2.945} ; \mathrm{R}^{2}=\right.$ 0.97). El índice de condición varió a lo largo del año (Fig. 6A) con valores máximos en julio (2009) y febrero, y los menores en septiembre (2009), junio y julio (2010); se encontró diferencias significativas en los valores de $\mathrm{K}$ entre meses $\left(\mathrm{F}_{(1.699)}=33.14, \mathrm{p}<0.0001\right)$ y entre sexos para enero $\left(\mathrm{F}_{(3.972)}=8.57, \mathrm{p}<0.0001\right)$, febrero $\left(\mathrm{F}_{(4.098)}=9.85, \mathrm{p}<0.0001\right)$ y mayo $\left(\mathrm{F}_{(3.981)}=\right.$ $9.02, \mathrm{p}<0.0001)$.
Para C. ensiferus no se encontró diferencia significativa en la relación talla-peso entre machos y hembras $(\mathrm{t}=0.34$, g.l. $=2$ 021, $\mathrm{p}>$ 0.05), y quedó establecida la relación de tipo alométrico positivo: $\mathrm{P}=0.0049 * \mathrm{~L}^{3.235}\left(\mathrm{R}^{2}=\right.$ 0.91). El índice de condición también varió a lo largo del periodo de estudio; se encontró en los meses de julio y agosto (2009) y febrero los valores más altos, y los más bajos durante junio y julio (2010). Se evidenciaron diferencias significativas en los valores de $\mathrm{K}$ entre los meses $\left(\mathrm{F}_{(1.697)}=64.36, \mathrm{p}<0.0001\right) \mathrm{y}$ entre sexos para los meses de septiembre $\left(\mathrm{F}_{(3.874)}=5.70, \mathrm{p}<\right.$ $0.0001)$, enero $\left(F_{(3.902)}=18.64, p<0.0001\right)$, 


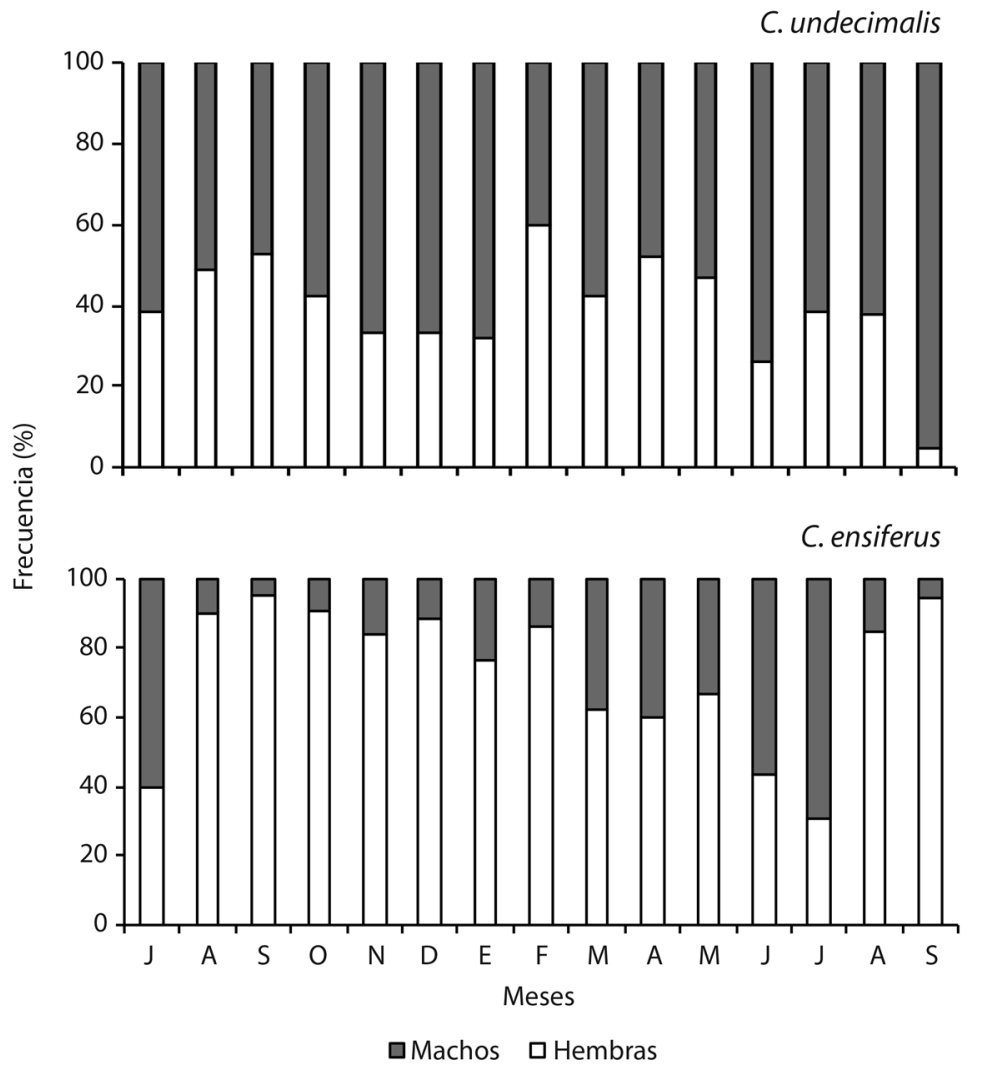

Fig. 2. Variación mensual del porcentaje de hembras y machos de C. undecimalis y C. ensiferus de julio 2009 a septiembre 2010 en la Laguna de Tacarigua, Venezuela.

Fig. 2. Monthly sex ratio of C. undecimalis y C. ensiferus of July 2009 to September 2010 in the Laguna de Tacarigua, Venezuela.

marzo $\left(\mathrm{F}_{(3.965)}=7.96, \mathrm{p}<0.0001\right)$ y abril $\left(\mathrm{F}_{(3.873)}=15.51, \mathrm{p}<0.0001\right)$.

\section{DISCUSIÓN}

El rango de tallas encontrado para $C$. undecimalis fue similar a lo reportado para la especie en otras áreas del Golfo de México, en Florida y el Lago de Nicaragua, que oscilan entre 12.4 y $114 \mathrm{~cm}$ (Taylor et al., 2000; Camacho \& Gadea, 2005; Caballero, 2009; Blewett, Stevens, Champeau, \& Taylor, 2009; Perera-García, Mendoza, Contreras, Huerta, \& Pérez, 2011; Lorán-Núñez, Martínez, Valdez, Garduño, \& Martínez, 2012; Perera-García et al., 2013; Andrade et al., 2013; Hernández,
Chiappa, \& Contreras, 2014). Para C. ensiferus fue similar a las tallas señaladas por Camacho \& Gadea (2005) para el Lago de Nicaragua (25 a $48 \mathrm{~cm}$ ) y Pérez, Álvarez, Mojica, y Dix (2005) quienes reportan como talla máxima $49.6 \mathrm{~cm}$. Para ambas especies las hembras alcanzan y dominan las tallas más grandes como ha sido previamente reportado (Taylor et al., 2000; Camacho \& Gadea, 2005; Perera-García et al., 2011; Andrade et al., 2013; Hernández et al., 2014).

La proporción sexual en C. undecimalis fue mayor para los machos (1:1.5), esto concuerda con los antecedentes previos para la especie. Taylor et al. (2000) encontraron proporciones de $1: 1.8$ y $1: 1.3$ y Perera-García et al. (2011) 


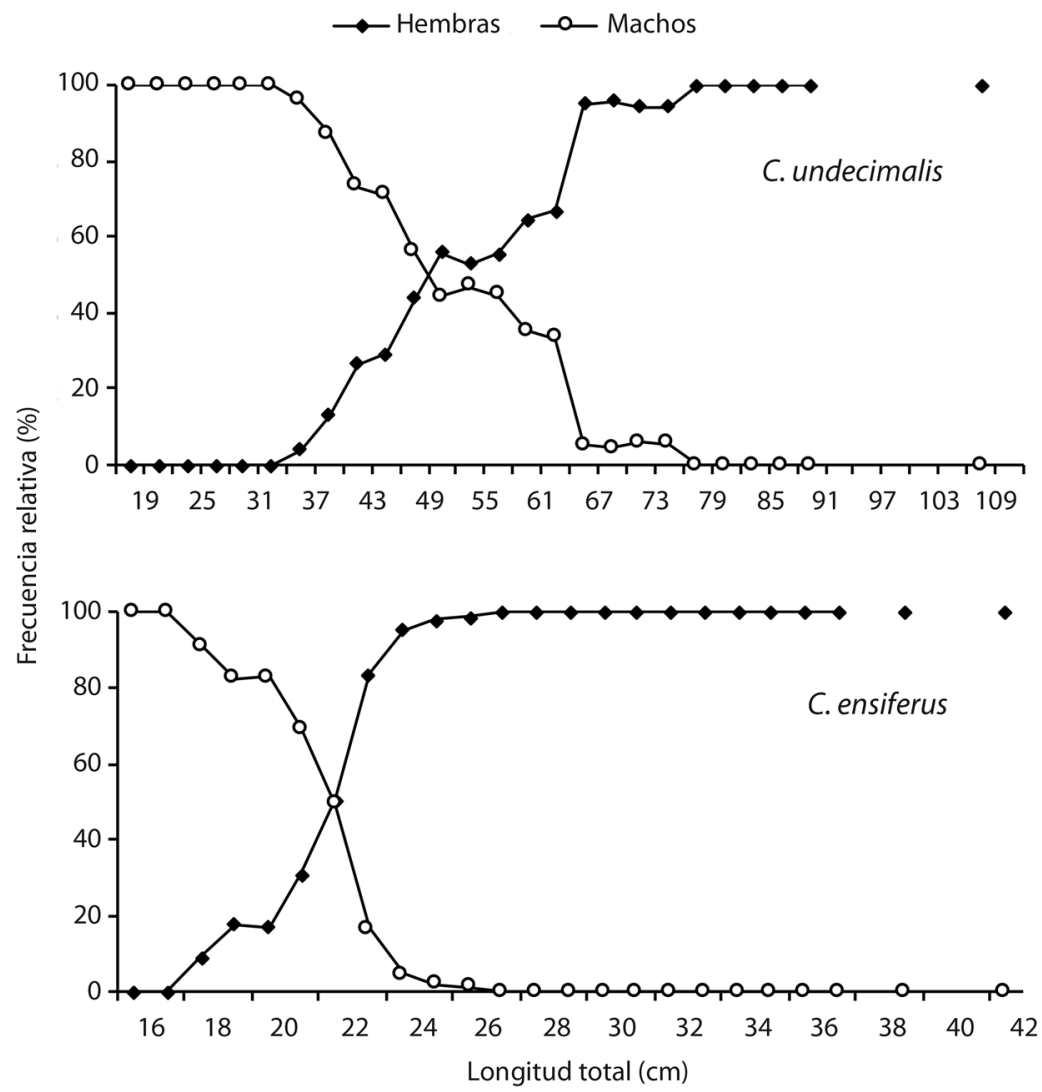

Fig. 3. Proporción sexual de machos y hembras por rango de tallas de C. undecimalis y C. ensiferus en la Laguna de Tacarigua, Venezuela.

Fig. 3. Sex ratio by length class of C. undecimalis y C. ensiferus in the Laguna de Tacarigua, Venezuela.

1:1.46 con meses de predominancia de machos hasta el $93 \%$, similar al presente estudio. Andrade et al. (2013) reportaron 1:1.5 con una amplia variación estacional y periódica, mientras que Hernández et al. (2014) encontraron $1: 3$ con variaciones en ambientes dulceacuícola (1:7.6) y marino (1:1.5). En el caso de $C$. ensiferus, la proporción sexual fue dominada por las hembras (1:3.3), con importantes variaciones estacionales, coincidiendo con lo reportado para el Lago de Nicaragua, donde se observó una proporción de 12 hembras por cada macho (Camacho \& Gadea, 2005). Estas importantes variaciones mensuales en la proporción sexual podrían responder no solo a agregaciones locales de hembras o emigraciones de machos, sino también a eventos de cambio de sexo como ha sido reportado para $C$. undecimalis (Andrade et al., 2013).

Lo anterior se pone de manifiesto al observar la proporción sexual a la talla, la cual evidenció diferencias significativas para las tallas menores y mayores en ambas especies. En $C$. undecimalis, se observó la predominancia de individuos machos en las tallas menores a 46 $\mathrm{cm}$ y de las hembras en tallas superiores a 67 $\mathrm{cm}$. Mientras en $C$. ensiferus, la predominancia de individuos machos en tallas menores a 20 $\mathrm{cm}$ y de las hembras en superiores a $23 \mathrm{~cm}$. Esta es una característica propia de especies que presentan hermafroditismo protándrico, como ha sido descrito para C. undecimalis, 


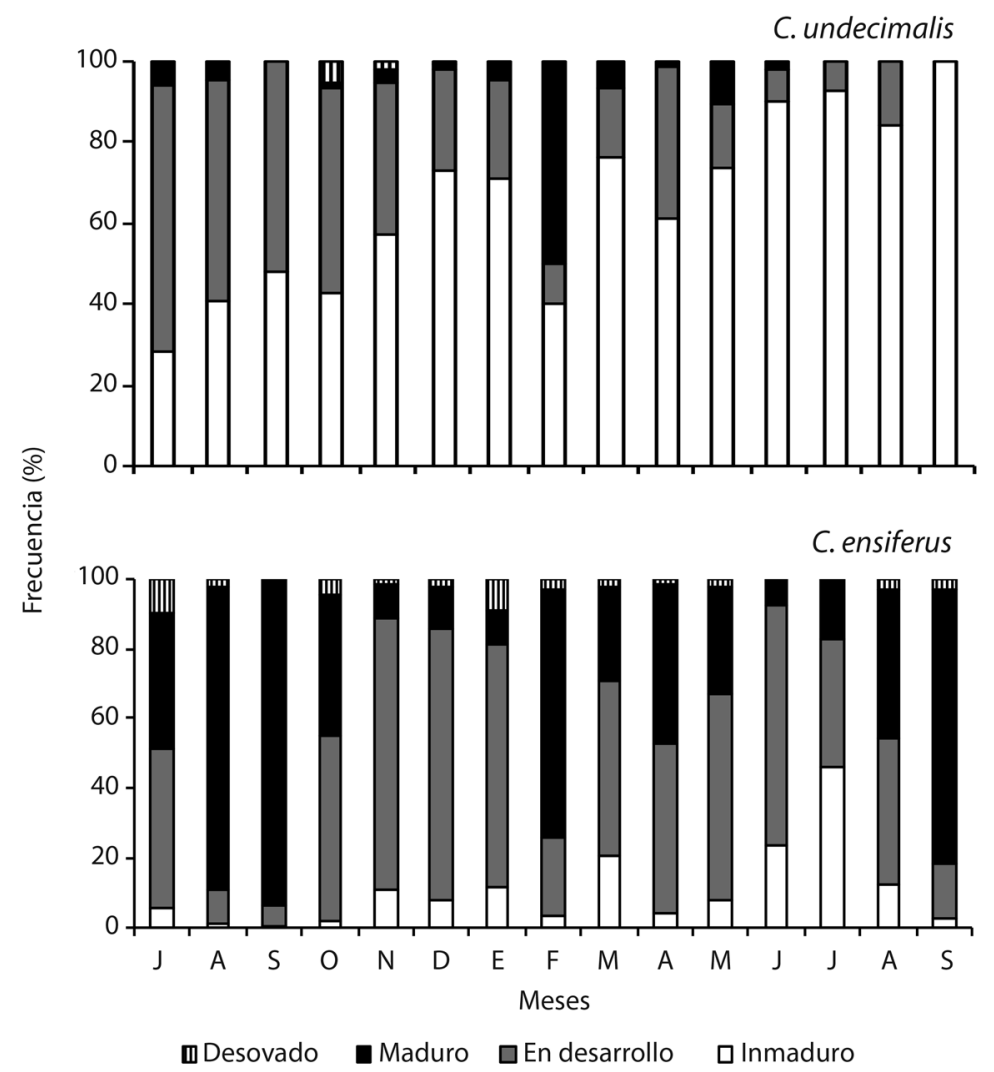

Fig. 4. Variación mensual de la frecuencia relativa de las fases de madurez sexual de hembras y machos de C. undecimalis y C. ensiferus de julio 2009 a septiembre 2010 en la Laguna de Tacarigua, Venezuela.

Fig. 4. Monthly variation of relative frequency maturity stages of females and males of C. undecimalis y C. ensiferus of July 2009 to September 2010 in the Laguna de Tacarigua, Venezuela.

en el cual la mayoría de los individuos jóvenes son machos y a partir de un determinado tamaño inician una reversión sexual, llegando a encontrarse, simultáneamente en las gónadas, lamelas ovígeras y remanentes de espermaductos dorsales con esperma (Peters et al., 1998; Taylor et al., 2000). Perera-García et al. (2011) encontraron diferencias en las proporciones sexuales a la talla en $C$. undecimalis, salvo en los rangos alrededor de 70 a $90 \mathrm{~cm}$, sugiriendo este como el rango de reversión sexual, aunque Andrade et al. (2013) indican que dicha reversión ocurre en promedio a una longitud total de $74.7 \mathrm{~cm}$ (6 a 8 años de edad), algo mayor a la encontrada en el presente trabajo $(47 \mathrm{a} 68 \mathrm{~cm})$, pero coincidente con lo señalado por Taylor et al. (2000) de una media de $51.5 \mathrm{~cm}$ (3.4 años), lo cual podría estar asociado a las zonas de estudio como reportan Hernández et al. (2014) quienes señalan un rango de talla menor para la reversión sexual en un área dulceacuícola con respecto a una marina. Grier \& Taylor (1998) señalan que casi el $100 \%$ de los ejemplares de $C$. undecimalis son susceptibles al cambio de sexo, al observar que los peces más grandes y viejos de estas poblaciones son hembras. Lo cual estimula a que los machos más jóvenes experimenten el proceso de reversión sexual, el cual tarda de 60 a 90 días y ocurre posterior a la época reproductiva (Peters et al., 1998). 


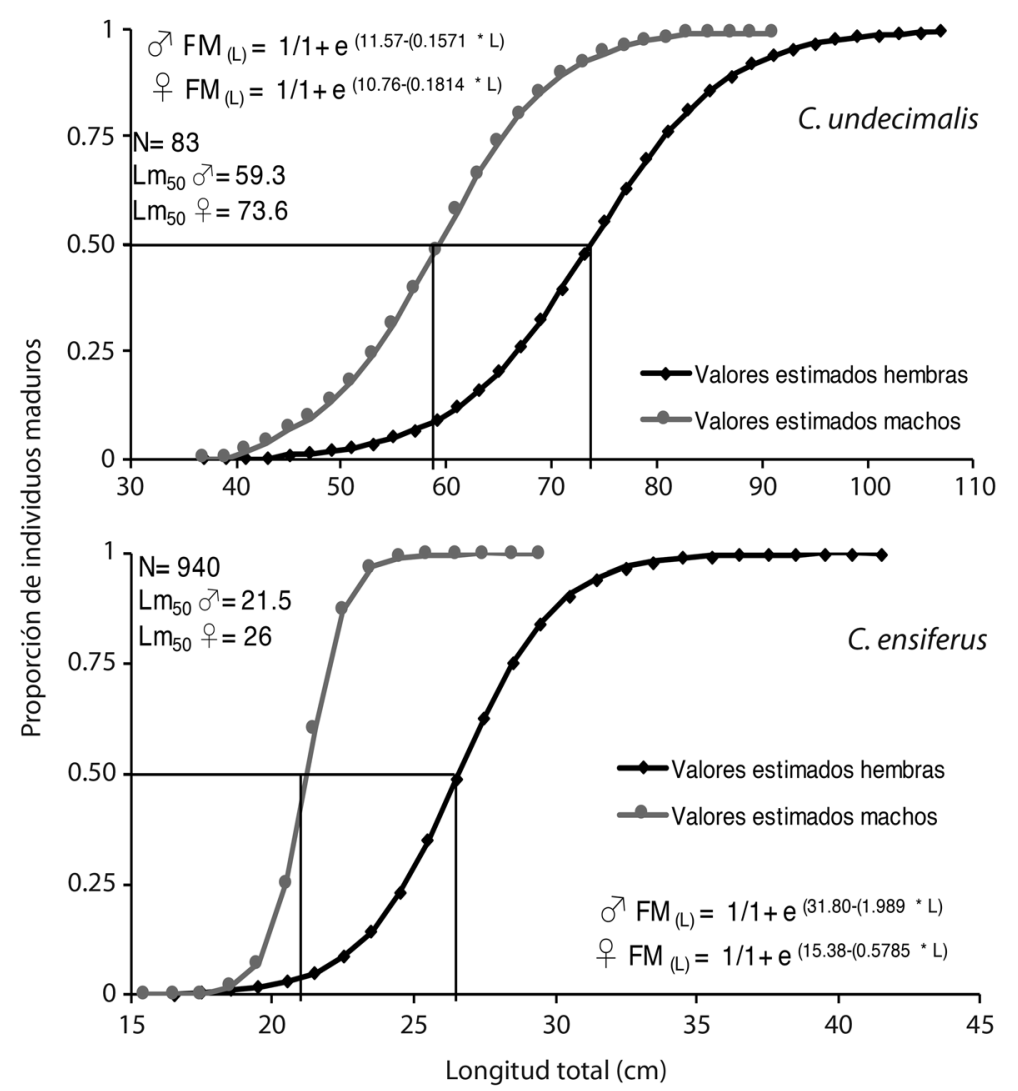

Fig. 5. Talla media de madurez sexual de la población $\left(\mathrm{L}_{50}\right)$ de hembras y machos de C. undecimalis y C. ensiferus en la Laguna de Tacarigua, Venezuela.

Fig. 5. Sexual maturity curve and size at maturity $\left(\mathrm{L}_{50}\right)$ of females and males of C. undecimalis y C. ensiferus in the Laguna de Tacarigua, Venezuela.

El desove de estas especies es asincrónico, lo cual causa un desfase en la época de reproducción, con muchos individuos con un desove parcial característico de regiones tropicales (Perera-García et al., 2011; Lowerre-Barbieri, Vose, \& Whittington, 2003), y puede ocurrir la mayor parte de los meses del año con períodos cortos de mayor intensidad (Camacho \& Gadea, 2005); aunque Peters et al. (1998) señalan que C. undecimalis presenta un desarrollo sincrónico de los ovarios. La escasa presencia de individuos maduros, en desove o desovados de C. undecimalis, durante el periodo de estudio, indicaría que la Laguna de Tacarigua es principalmente un área de crecimiento y alimentación. Márquez (1985) señala que la especie no cumple su ciclo reproductivo completo dentro de la Laguna, ya que migra a mar abierto a desovar. Blewett et al. (2009) sugieren que algunos individuos migran a zonas costeras para reproducirse y retornan a los estuarios. Casi la totalidad de los ejemplares maduros en el presente estudio, se encontraron durante el mes de febrero, señalando este periodo como el de máxima actividad reproductiva. Es conveniente destacar que durante ese periodo la boca de la laguna se encontraba cerrada, por lo que no existía contacto de la laguna con el mar, por lo cual la captura de estos ejemplares maduros y desovados, pudo deberse a su imposibilidad 


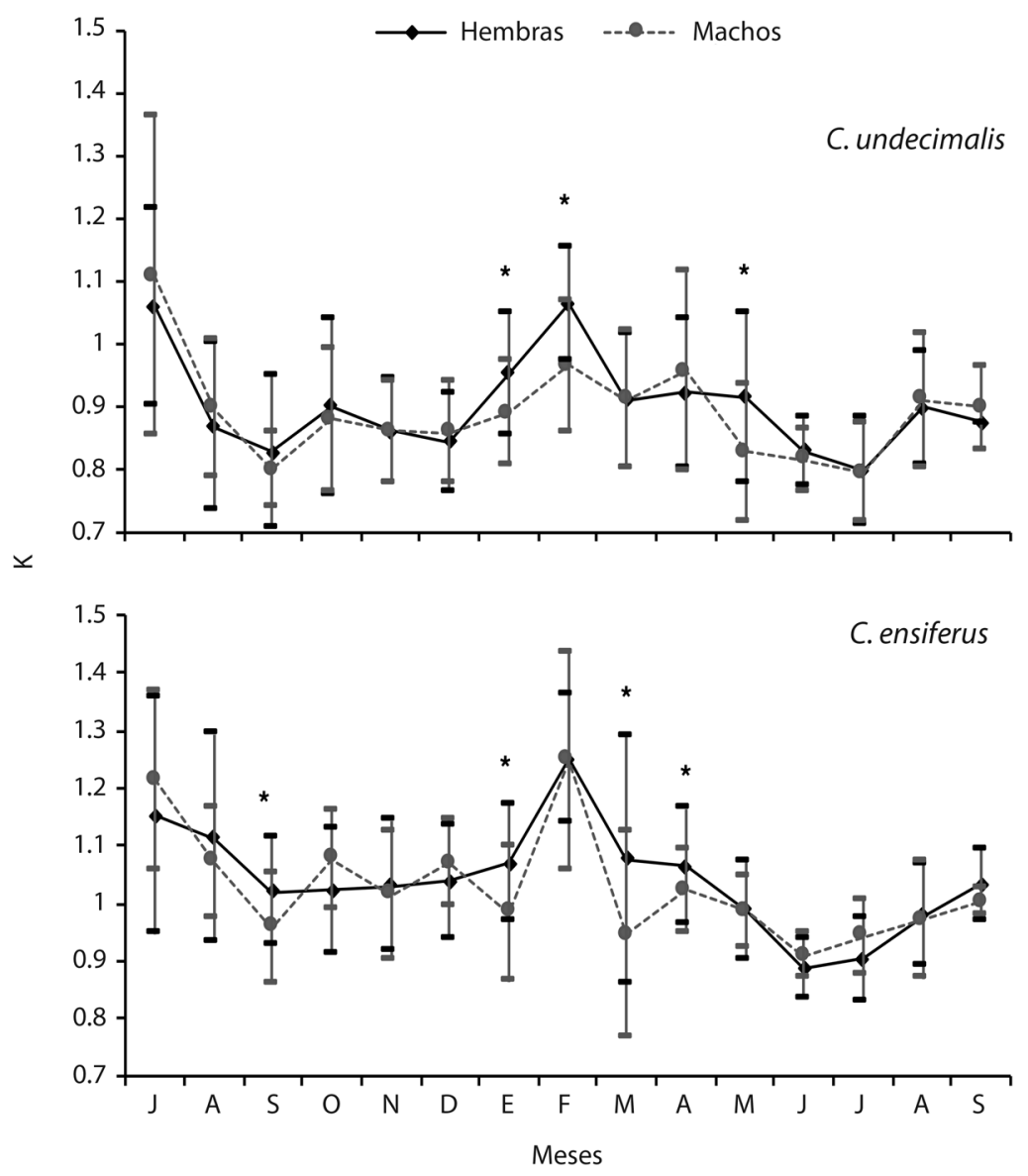

Fig. 6. Factor de condición de hembras y machos de C. undecimalis y C. ensiferus de julio 2009 a septiembre 2010 en la Laguna de Tacarigua, Venezuela (Promedio y desviación estándar). *Diferencias significativas entre sexos.

Fig. 6. Condition Factor for females and males of C. undecimalis y C. ensiferus of July 2009 to September 2010 in the Laguna de Tacarigua, Venezuela (mean and standard deviation). *Significant differences between sexes.

de salir a desovar al mar y por ello realizarlo en la laguna. Se ha demostrado que individuos adultos que permanecen en ambientes costeros o dulceacuícolas pueden alcanzar la madurez sexual (Hernández et al., 2014). Perera-García et al. (2011) afirman que la reproducción solo se presentó en áreas costeras, y ésta fue de abril a septiembre, con un pico entre los meses de julio, agosto y septiembre, los cuales estuvieron relacionados con temperaturas del agua de entre 26 a $30{ }^{\circ} \mathrm{C}$, similares a las encontradas en el presente estudio. Caballero (2009) en Campeche, señala una temporada de reproducción de marzo a octubre, mientras Peters et al. (1998) indican que en Florida el periodo reproductivo es prolongado, de abril a diciembre o enero, con un mayor pico entre mayo y septiembre, lo que coincide con lo reportado en el sistema lagunar de Alvarado por Galaviz, Cervantes, \& Rodríguez (2006), aunque LoránNúñez et al. (2012) señalan un periodo de junio a septiembre en el mismo sistema. Chapman, Horel, Fish, Jones, \& Spicola (1978) sugieren además que el proceso requiere de altas salinidades (mayor a $28 \mathrm{ppm}$ ) para la fertilización de sus huevos, aunque las mayores registradas 
aquí no superaron las 24.3 ppm. Cervigón (1991) señala que en la región nororiental de Venezuela, $C$. undecimalis se reproduce entre abril y septiembre, con dos meses de mayor intensidad: mayo y septiembre. Gilmore, Donahoe, \& Cooke (1983) encontraron además que existe una correlación positiva entre la actividad de desove y los meses lluviosos, pero no así con la temperatura y la salinidad.

Se encontraron individuos de C. ensiferus maduros y desovados durante todos los meses de estudio, indicando que la especie se reproduce durante todo el año, con dos periodos de mayor intensidad reproductiva, el más importante entre agosto y septiembre y el segundo de febrero a abril. Estos resultados concuerdan con Márquez (1985), quien señala que la especie se reproduce de abril a septiembre, presentando máximos de actividad reproductiva en abril-mayo y septiembre. Por su parte, Sánchez (2001) reportó individuos próximos al desove en marzo, abril y mayo en la desembocadura del río Grande y Laguna de Perlas (Mar Caribe de Nicaragua), mientras Keith et al. (2000) indicaron que la especie se reproduce de abril a septiembre. Cervigón (1991) por su parte, señala que la especie puede cumplir todo su ciclo vital en el interior de las lagunas litorales costeras. Es conveniente resaltar, para ambas especies, que los meses de mayor presencia de individuos maduros, coinciden con los de mayor proporción de hembras. En C. ensiferus los meses de agosto a octubre (2009), febrero y agosto y septiembre (2010), así como para $C$. undecimalis el mes de febrero, lo cual pudiera estar relacionado con periodos de reversión sexual o emigraciones de machos como ha sido previamente señalado (Adams et al., 2009; Andrade et al., 2013), lo cual también estaría relacionado a las importantes variaciones mensuales en la abundancia de la especie en la zona (Gassman \& López, 2015b).

La talla de madurez sexual $\left(\mathrm{Lm}_{50}\right)$ estimada para $C$. undecimalis en el presente trabajo ( $($ : $: 73.65 \mathrm{y}$ o $: 59.3 \mathrm{~cm}$ ), se encuentra por debajo de lo reportado para la especie en el Golfo de México por Perera-García et al. (2011) quienes señalan tallas de $80 \mathrm{~cm}$ para hembras y $60 \mathrm{~cm}$ para machos, mientras Hernández et al. (2014) reportan de 78.7 y $80 \mathrm{~cm}$ para machos y 85 y $89 \mathrm{~cm}$ para hembras. No así, por lo señalado por Peters et al. (1998) quienes determinaron de 50 a $52.2 \mathrm{~cm}$ para hembras y de 33 a 34.8 $\mathrm{cm}$ para machos (longitud estándar) en Florida y Ospina, Pardo, \& Álvarez (2008) quienes reportaron que el único macho capturado en estado maduro tenía $47 \mathrm{~cm}$ de longitud estándar. Por su parte, Taylor et al. (2000) manifiestan que los machos pueden alcanzar la madurez sexual en el primer año, con tallas entra 15 y 20 $\mathrm{cm}$ (Longitud furcal) y algunos se desarrollan a hembras entre 1 y 7 años de edad con tallas de entre 24 a $82.4 \mathrm{~cm}$. Es importante destacar que el $87 \%$ de los individuos capturados en la Laguna de Tacarigua, se encontraron por debajo de la talla media de madurez sexual. Para C. ensiferus la talla de madurez $\operatorname{Lm}_{50}(+$ : 26 y ${ }^{\lambda}: 21.5 \mathrm{~cm}$ ) fue similar a lo reportado por Márquez (1985) para la Laguna de Tacarigua de $25.2 \mathrm{~cm}$ (longitud estándar). En este caso, el $39.4 \%$ de los individuos capturados, se encontraron por debajo de la talla media de madurez sexual. Para ambas especies, ésta es una señal de alerta por los efectos que esto podría conllevar para la adecuada renovación de sus poblaciones, debido a una probable sobrepesca por crecimiento en el área.

El tipo de crecimiento de $C$. undecimalis fue diferente para hembras y machos, en el primero se evidenció un crecimiento alométrico positivo, mientras para los segundos fue isométrico, al igual que para toda la población en conjunto. Este tipo de crecimiento también fue observado para juveniles de la laguna de $\mathrm{La}$ Restinga (Marcano, 1976) y por numerosos trabajos en el Golfo de México y aguas de Florida (Carvajal, 1975; Taylor et al., 2000; Galaviz et al., 2006; Caballero, 2009; Perera-García et al., 2013; Hernández et al., 2014). En el caso de $C$. ensiferus se evidenció un crecimiento alométrico positivo para ambos sexos, lo cual contrasta con lo reportado por Bouchon-Navaro, Bouchon, Kopp \& Louis (2006) que indican un crecimiento isométrico para esta especie.

El factor de condición (K), presentó variaciones mensuales significativas para ambas 
especies. Este índice tiende a variar de acuerdo a factores como la disponibilidad de alimento, el sexo, la estacionalidad y el grado de desarrollo gonadal (Froese, 2006). Para ambas especies se encontraron los mayores valores de $\mathrm{K}$ en los meses de mayor actividad reproductiva, en el caso de $C$. undecimalis en julio (2009) y febrero, mientras en $C$. ensiferus en julio y agosto (2009) y febrero, sugiriendo que esos altos valores pudieran estar asociados a la época reproductiva, como señalan Andrade et al. (2013), y su decrecimiento en los meses subsiguientes debido a eventos de desove.

Se concluye que ambas especies presentan un patrón típico de hermafroditismo protándrico, con actividad reproductiva durante todo el año para $C$. ensiferus, y en el primer trimestre para C. undecimalis, evidenciándose además, altos porcentajes de individuos por debajo de la talla media de madurez sexual en las capturas. En este sentido, considerando que se ha reportado una importante disminución de los recursos pesqueros en el zona (Gassman \& López, 2015b), se recomienda como medidas de manejo para estas especies, el establecimiento de un periodo de veda (durante el primer trimestre del año) y de tallas mínimas de captura, en donde se considere el carácter de hermafroditismo protándrico y por consiguiente esta sea posterior al inicio de la reversión sexual, es decir, al menos $74 \mathrm{~cm}$ para $C$. undecimalis y $26 \mathrm{~cm}$ para C. ensiferus. Es necesario, además, enfocar futuros estudios en la zona costera adyacente, a fin de evaluar las migraciones reproductivas y zonas de desove, considerando que se ha señalado un marcado patrón de fidelidad a localidades o áreas reproductivas, lo cual conlleva importantes implicaciones ecológicas (Adams et al., 2009; Lowerre-Barbieri et al., 2014), que deben tomarse en cuenta para el manejo y la gestión integral de la estructura espacial de conectividad entre los lugares de desove, áreas de transporte de larvas y hábitats de crecimiento para estas especies.

\section{AGRADECIMIENTOS}

A la comunidad de pescadores de la Laguna de Tacarigua por su amabilidad y colaboración, especialmente a Carlos Barboza. A los revisores anónimos por sus valiosas recomendaciones.

\section{RESUMEN}

Los róbalos (C. undecimalis y $C$. ensiferus) son recursos pesqueros de importancia en toda su área de distribución. En Venezuela son capturados por pesquerías artesanales a lo largo de toda la costa, sin embargo, los estudios sobre su biología que permitan orientar regulaciones de su pesca son escasos. Para analizar aspectos de la reproducción de ambas especies se analizaron 1325 individuos de C. undecimalis y 2025 de $C$. ensiferus, provenientes de la pesca artesanal en la Laguna de Tacarigua, en el norte de Venezuela, desde julio 2009 a septiembre 2010. Se midió la longitud total, peso total, sexo y madurez sexual de cada individuo. La proporción sexual varió a lo largo del año, se observó una diferencia significativa en favor de los machos para $C$. undecimalis $(1: 1.5)$ y de las hembras para $C$. ensiferus (1:3.3). Se evidenciaron diferencias en la proporción sexual de la talla para ambas especies: los machos dominan las tallas inferiores, mientras que las hembras dominan las superiores. Lo cual señala un patrón típico de hermafroditismo secuencial (protándrico) en ambas. Se estimaron las tallas de madurez sexual $\left(\mathrm{Lm}_{50}\right)$ para $C$. undecimalis $(q=$ $73.6 \mathrm{y} \hat{\delta}=59.3 \mathrm{~cm})$ y $C$. ensiferus $(\uparrow=26 \mathrm{y} \hat{\jmath}=21.5 \mathrm{~cm}) . C$. ensiferus se reproduce durante todo el año con dos periodos de mayor intensidad (agosto a septiembre y febrero a abril), mientras para C. undecimalis fueron escasos los individuos maduros, mostrando febrero el pico de reproducción más importante. Se encontró diferencia en el crecimiento entre machos y hembras de $C$. undecimalis: alométrico positivo para hembras $\left(\mathrm{P}=0.0022 * \mathrm{~L}^{3.353}\right)$ y negativo para machos $\left(\mathrm{P}=0.0108 * \mathrm{~L}^{2.945}\right)$, mientras no se encontraron diferencias para $C$. ensiferus, que presentó alometría positiva $(\mathrm{P}=$ $0.0049 * \mathrm{~L}^{3.235}$ ) para ambos sexos. La falta de regulación y controles de pesca, la evidencia de importantes volúmenes de individuos inmaduros en las capturas, así como el carácter particular de la biología reproductiva de estas especies, sugiere la necesidad de establecer regulaciones para garantizar su aprovechamiento responsable, como un periodo de veda durante el primer trimestre de año y tallas mínimas de captura para ambas especies.

Palabras clave: hermafroditismo protándrico, Centropomus, róbalo, Laguna de Tacarigua. 


\section{REFERENCIAS}

Adams, A., Wolfe, R., Barkowski, N., \& Overcash, D. (2009). Fidelity to spawning grounds by a catadromous fish, Centropomus undecimalis. Marine Ecology Progress Series, 389, 213-222.

Andrade, H., Santos, J., \& Taylor, R. (2013). Life-history traits of the common snook Centropomus undecimalis in a Caribbean estuary and large-scale biogeographic patterns relevant to management. Journal of Fish Biology, 82, 1951-1974.

Blewett, D., Stevens, P., Champeau, T., \& Taylor, R. (2009). Use of rivers by common snook Centropomus undecimalis in southwest Florida: a first step in addressing the overwintering paradigm. Biological Sciences, 72, 310-324.

Bouchon-Navaro, Y., Bouchon, C., Kopp, D., \& Louis, M. (2006). Weight-length relationships for 50 fish species collected in sea grass beds of the Lesser Antilles. Journal of Applied Ichthyology, 22, 322-324.

Caballero, V. (2009). Pesquería de róbalo blanco Centropomus undecimalis en Campeche, México. Ciencia Pesquera, 17, 77-86.

Camacho, J., \& Gadea, V. (2005). Estudio técnico científico del róbalo en Río San Juan y el Gran Lago de Nicaragua. Managua: Marena.

Carvajal, R. (1975). Contribución al conocimiento de la biología de los róbalos Centropomus undecimalis y C. poeyi en la laguna de Términos, Campeche, México. Boletín de Instituto Oceanográfico de Venezuela, 14, 51-71.

Cervigón, F. (1991). Los Peces Marinos de Venezuela. Caracas: Fundación Científica de Los Roques.

Cervigón, F., Cipriani, R., Fischer, W., Garibaldi, I., Hendrickx, M., Lemus, A., Márquez, R., Poutiers, J., Robaina, G., \& Rodríguez, B. (1992). Guía de campo de las especies comerciales marinas y de aguas salobres de la costa septentrional de Sur América. Roma: FAO.

Chao, L., Mendonça, J., Albieri, R., Giarrizzo, T., Fadré, N., da Silva, F., Brick, M., Castro, M., Villwock, L., \& Vieira, J. (2015). Centropomus ensiferus. The IUCN Red List of Threatened Species 2015: e.T191831A2007019.

Chapman, P., Horel, G., Fish, W., Jones, K., \& Spicola, J. (1978). Artificial culture of snook, Rookery Bay, 1977. Induced spawning and fry culture (Annual Report on Sportfish Introductions). USA: Florida Game and Fresh Water Fish Commission.

Froese, R. (2004). Keep it simple: three indicators to deal with overfishing. Fish and Fisheries, 5, 86-91. DOI:10.1111/j.1467-2979.2004.00144.x
Froese, R. (2006). Cube law, condition factor and weightlength relationships: history, meta-analysis and recommendations. Journal of Applied Ichthyology, 22, 241-253.

Gaertner, D., \& Laloé, F. (1986). Etude biométrique de la taille á première maturité sexuelle de Geryon maritae Manning et Holthuis, 1981 du Sènegàl. Oceanologica Acta, 9, 479-487.

Galaviz, V., Cervantes, M., \& Rodríguez, M. (2006). Biología pesquera del róbalo Centropomus undecimalis en el sistema lagunar de Alvarado, Veracruz. Simposio sobre ecosistemas costeros del Golfo de México y Mar Caribe, Villahermosa, México.

Gassman, J., \& López, H. (2015a). Dinámica poblacional y explotación de la mojarra Eugerres plumieri en el Parque Nacional Laguna de Tacarigua, Venezuela. Ciencia, 23, 11-20.

Gassman, J., \& López, H. (2015b). Variación de la abundancia de los recursos pesqueros en el parque nacional Laguna de Tacarigua, Venezuela. Acta Biológica Venezuelica, 35, 11-25.

Gilmore, R., Donahoe, C., \& Cooke, D. (1983). Observations on the distribution and biology of east-central Florida population of the common snook, Centropomus undecimalis (Bloch). Florida Scientist, 46, 313-336.

Grier, H., \& Taylor, R. (1998). Testicular maturation and regression in the common snook. Journal of Fish Biology, 3, 521-542.

Hernández, U., Chiappa, X., \& Contreras, W. (2014). Reproductive variability of the common snook, Centropomus undecimalis, in environments of contrasting salinities interconnected by the Grijalva-Usumacinta fluvial system. Ciencias Marinas, 40, 173-185.

Hoydal, K., Rorvik, C., \& Sparre, P. (1982). Estimation of effective mesh sizes and their utilization in assessment. Dana, 2, 69-95.

Jennings, S., Kaiser, M., \& Reynolds, J. (2001). Marine Fisheries Ecology. Oxford: Blackwell.

Keith, P., Le Bail, P., \& Planquette, P. (2000). Atlas des poissons d'eau douce de Guyane Paris: Publications scientifiques du Muséum national d'Histoire naturelle.

Lorán-Núñez, R., Martínez, F., Valdez, A., Garduño, M., \& Martínez, E. (2012). Reproducción y madurez sexual del róbalo prieto (Centropomus poeyi) y róbalo blanco (C. undecimalis) en el Sistema Lagunar de Alvarado, Veracruz (2005-2007). Ciencia Pesquera, 20, 49-64.

Lowerre-Barbieri, S., Vose, F., \& Whittington, J. (2003). Catch and release fishing on a spawning aggregation of common snook: does it affect reproductive output. 
Transactions of the American Fisheries Society, 132, 940-952.

Lowerre-Barbieri, S., Villegas-Rios, D., Walters, S., Bickford, J., Cooper, W., Muller, R., \& Trotter, A. (2014). Spawning Site Selection and Contingent Behavior in Common Snook, Centropomus undecimalis. PLoS ONE, 9(7), e101809. doi:10.1371/ journal.pone.0101809

Marcano, J. (1976). Aspectos biológicos de los juveniles de Centropomus undecimalis (Bloch, 1972) de la Laguna de La Restinga y sus alrededores, Estado Nueva Esparta (Pisces: Centropomidae) (Tesis de pregrado). Universidad de Oriente, Venezuela.

Márquez, P. (1985). Algunos aspectos de la biología del róbalo Centropomus ensiferus Poey, 1860 y C. undecimalis (Bloch, 1792) (Osteichthyes Centropomidae) en la Laguna de Tacarigua, Venezuela (Tesis de pregrado). Universidad Central de Venezuela, Venezuela.

Mendonça, J., Chao, L., Albieri, R., Giarrizzo, T., da Silva, F., Castro, M., Brick, M., Villwock, L., \& Vieira, J. (2015). Centropomus undecimalis. The IUCN Red List of Threatened Species 2015: e.T191835A200725.

Nikolsky, G. V. (1963). The ecology of fishes. London: Academic Press.

Novo, I., Morales, L., Rodríguez, C., Martínez, G., \& Hertelendy, I De. (1997). Ciencia y conservación en el sistema de Parque Nacionales de Venezuela: una experiencia de cooperación Interinstitucional. Caracas: Impresos Altamira.

Ospina, J., Pardo, F., \& Álvarez, R. (2008). Madurez gonadal de la ictiofauna presente en la Bahía de Cartagena, Caribe Colombiano. Boletín Científico del Museo de Historia Natural, 12, 117-140.

Perera-García, M., Mendoza, M., Contreras, W., Huerta, M., \& Pérez, E. (2011). Reproductive biology of common snook Centropomus undecimalis (Perciformes: Centropomidae) in two tropical habitats. Revista de Biología Tropical, 59, 669-681.
Perera-García, M., Mendoza, M., Contreras, W., Ferrara, A., Huerta, M., \& Hernández, R. (2013). Comparative age and growth of common snook Centropomus undecimalis (Pisces: Centropomidae) from coastal and riverine areas in Southern Mexico. Revista de Biología Tropical, 61, 807-819.

Pérez, L., Álvarez, M., Mojica, A., \& Dix, M. (2005). La ictiofauna del Refugio de Vida Silvestre Bocas del Polochic y la cuenca del lago de Izabal: composición, distribución y ecología. Ciudad de Guatemala: UNESCO.

Peters, M., Matheson, R., \& Taylor, R. (1998). Reproduction and early life history of common snook, Centropomus undecimalis (Bloch), in Florida. Bulletin of Marine Science, 62, 509-529.

Ramírez, P. (1996). Lagunas costaneras venezolanas. Porlamar: Benavente \& Martínez.

Ricker, W. (1975). Computation and interpretation of biological statistics of fish populations. Bulletin Fisheries Research Board of Canada, 191, 1-382.

Rivas, L. (1986). Systematic review of the perciform fishes of the genus Centropomus. Copeia, 3, 579-611.

Sánchez, R. (2001). Biología Pesquera de los Róbalos Género Centropomus en Lagunas Costeras de la Región Autónoma Atlántico Sur, Nicaragua. Managua: DIPAL.

Taylor, R., Grier, H., \& Whittington, J. (1998). Spawning rhythms of common snook in Florida. Journal of Fish Biology, 53, 502-520.

Taylor, R., Whittington, J., Grier, H., \& Crabtree, R. (2000). Age, growth, maturation, and protandric sex reversal in common snook, Centropomus undecimalis, from the east and west coasts of South Florida. Fishery Bulletin, 98, 612-624.

Zar, J. (1996). Biostatical analysis. Nueva Jersey: Prentice Hall. 